\title{
NuEVAS ESTRATEgIAS PARA LA ELABORACIÓN DE TFG EN INGLÉS: TUTORÍAS ENTRE IGUALES Y MINI-VÍDEOS DOCENTES
}

\author{
New Strategies for Writing Degree Dissertations \\ IN ENgLish: PAIR-Tutoring AND TEACHING MINI-VIDEOS
}

\author{
Cristina A. Huertas Abril, M. ${ }^{a}$ Elena Gómez Parra, \\ Ángela $\mathrm{M}^{\mathrm{a}}$ Larrea Espinar, Antonio R. Raigón Rodríguez, \\ David Bullejos Martín, Francisco Villamandos de la Torre, \\ Joaquín Olivares Bueno, José Manuel Palomares Muñoz, \\ $\mathrm{M}^{\mathrm{a}}$ Amalia Trillo Holgado, $\mathrm{M}^{\mathrm{a}}$ del Mar Delgado Serrano, \\ Magdalena López Reifs \\ cristina.huertas@uco.es \\ Universidad de Córdoba
}

Received: 30/07/2017_Accepted: 04/12/2017

\begin{abstract}
Writing academic texts in English is closely related to the linguistic competence that university students need to develop (B1 level as a minimum linguistic requirement prior to their graduation). This competence entails an improvement of their future employability (both at the national and international levels), their communicative competences in a second language (L2), and obviously also the possibility that the results derived from their academic projects spill across national boundaries. This innovation project arises as the evolution of a previous innovation strategy, approved and implemented at the University of Córdoba ("La tutoría piramidal como estrategia para el diseño y desarrollo del trabajo final de grado en educación", 2015-2-6011), whose results allowed the identification of the weaknesses that we aim to focus on during the development of this project. The final objective is to teach university students how to write their end-of-degree dissertations in English. At the same time teachers of the UCO also participate in a series of seminars to know different techniques about how to review these projects that are written in a language different from their mother tongue or main language. The methodology of this project is based on participative seminars, as well as on the development of resources, where students (mentors and mentees) and teachers (mentors and mentees) collaborate towards a common objective: writing and reviewing end-of-degree dissertations, mainly written in English. Among these resources, it can be highlighted the design and elaboration of teaching mini-videos, with the participation of the teachers involved in the project, as well as the mentor students, in order to explain the key aspects to be considered when writing an end-ofdegree dissertation.
\end{abstract}

Keywords: End-of-Degree Dissertation; English for Academic Purposes; Peer Tutoring; Teaching Mini-Videos; Seminars. 


\section{Resumen}

La redacción de textos académicos en inglés está íntimamente relacionada con la competencia lingüística que el alumnado universitario ha de desarrollar (B1 como requisito lingǘstico mínimo previo a su graduación) y que, de manera lógica, está encadenada a la mejora de su empleabilidad (tanto a niveles nacionales como internacionales), de sus competencias comunicativas en segunda lengua y, por supuesto, la posibilidad de que los resultados de su trabajo académico trasciendan fronteras. El proyecto que aquí se presenta surge como evolución de un proyecto de innovación docente anterior, aprobado e implementado en la UCO ("La tutoría piramidal como estrategia para el diseño y desarrollo del trabajo final de grado en educación”, 2015-2-6011), y cuyo resultado ha permitido identificar la necesidad que con esta propuesta pretendemos cubrir. El objetivo final de este proyecto es enseñar al alumnado universitario a redactar sus TFG en inglés, al tiempo que el profesorado también participa en los seminarios, en los que se abordan también técnicas que faciliten la corrección de estos trabajos que se redactan en una lengua no materna o principal. La metodología se basa en seminarios participativos, así como en la elaboración de recursos, en los que alumnado (tutores y tutorizados) y profesorado (tutores y tutorizados) colaborarán para la consecución final del objetivo: la redacción y corrección de TFG, principalmente en lengua inglesa. Entre estos recursos, destacamos el diseño y la elaboración de minivídeos docentes modulares con la participación del profesorado implicado en el proyecto, así como del alumnado mentor, para explicar los aspectos clave de la elaboración de un TFG.

Palabras clave: TFG; Inglés con fines académicos; Tutoría entre iguales; Minivídeos docentes; Seminarios.

\section{INTRODUCCIÓN}

La redacción de textos académicos en inglés está íntimamente relacionada con la competencia lingüística que el alumnado universitario ha de desarrollar (B1 como requisito lingüístico mínimo previo a su graduación) y que, de manera lógica, está encadenada a la mejora de su empleabilidad (tanto a niveles nacionales como internacionales), la mejora de sus competencias comunicativas en segunda lengua y, por supuesto, la posibilidad de que los resultados de su trabajo académico trasciendan fronteras. El proyecto que aquí se presenta surge como evolución de un proyecto de innovación docente anterior, aprobado e implementado en la UCO ("La tutoría piramidal como estrategia para el diseño y desarrollo del trabajo final de grado en educación”, 2015-2-6011) y cuyo resultado ha permitido identificar la necesidad que con esta propuesta se pretende cubrir.

Existen ya grupos de alumnado de algunas Titulaciones de Grado de la UCO que han cursado itinerarios bilingües (por 
ejemplo, Grado en Educación Primaria) y que, por tanto, ya han tenido la experiencia de redactar sus TFG (total o parcialmente) en inglés. Debido a una multiplicidad de razones (entre las que destacamos el alto número de alumnos matriculados), el profesorado que ha tutorizado dichos Trabajos no siempre ha pertenecido a Áreas Lingüísticas (en este caso inglés como segunda lengua o como lengua extranjera) y hemos podido contrastar la dificultad que muchos de ellos han experimentado al tener que abordar la corrección de textos académicos en esta lengua.

Entendemos, por tanto, que dotar al profesorado y al alumnado de las herramientas esenciales que les permitan desenvolverse en esta tarea con cierta solvencia es fundamental para la calidad de los Títulos Universitarios de la UCO, ya que una de sus líneas estratégicas (y, por ende, prioritarias) es la implantación de Grados Bilingües, para lo que las habilidades de redacción (para el alumnado) y corrección (para el profesorado) de textos académicos y presentaciones en inglés es esencial.

\section{OBJETIVOS}

El objetivo esencial de este proyecto consiste en proporcionar al alumnado y profesorado universitarios las herramientas necesarias que les permitirán redactar y estructurar correctamente textos académicos en inglés (en especial, Trabajos Fin de Grado), así como elaborar y preparar sus presentaciones orales en dicha lengua. El carácter especial de los TFG viene derivado del hecho de que, a pesar de la equiparación legislativa con otras asignaturas (Gómez, Serrano y Huertas, 2015), el alumnado universitario lo considera como una dura prueba debido a varios factores de carácter normativo y académico, entre los que se destaca que han de demostrar que, en líneas generales, ha adquirido tanto los conocimientos propios de su profesión, como las competencias que la titulación ha definido para sus egresados, incluida la competencia genérica o transversal que implica el conocimiento de una lengua extranjera.

Las técnicas de estructura y organización de párrafos, la correcta composición de oraciones, la expresión de la lógica textual y la presentación del texto son básicas para el alumnado que, bien por estar cursando itinerarios bilingües o en segunda lengua, bien por tener la inquietud o necesidad de aprender a redactar textos académicos en inglés son las bases de este proyecto. Esto se complementa con el aprendizaje de las técnicas para la elaboración de una presentación en inglés y su defensa oral en público o ante un 
tribunal. De manera más concreta, se detallan los siguientes objetivos específicos:

1. Desarrollar las estrategias necesarias para la redacción y revisión de trabajos académicos redactados en lengua inglesa.

2. Conocer las herramientas y los recursos necesarios para redactar y estructurar correctamente textos académicos en inglés.

3. Aplicar estrategias para el desarrollo del trabajo en equipo.

4. Mejorar los resultados de aprendizaje del alumnado, especialmente aquellos relacionados con la lengua inglesa.

5. Diseñar y elaborar los Trabajos Fin de Grado en lengua inglesa.

6. Crear un repositorio de recursos para la elaboración de Trabajos de Fin de Grado.

7. Diseñar y elaborar minivídeos docentes modulares con la participación del profesorado implicado en el proyecto, así como del alumnado mentor, para explicar distintas partes de la elaboración de un TFG.

\section{METODOLOGÍA}

Para el desarrollo de este proyecto de innovación docente nos hemos basado en una metodología participativa y colaborativa a fin de desarrollar el inglés para fines académicos en el contexto de los trabajos de fin de grado. En este proyecto, el alumnado es el protagonista de su propio proceso de aprendizaje, fomentando el aprendizaje autónomo y gracias a un conjunto de herramientas que fomentan una comunicación individual y grupal, principalmente en lengua inglesa, generando contextos de aprendizaje positivos, y que facilitan y favorecen la relación educativa entre todos los participantes implicados.

\subsection{Participantes}

Este proyecto se ha desarrollado mediante seminarios participativos e interactivos en los que se han agrupado los cuatro colectivos anteriormente esbozados y que detallamos a continuación:

a. Profesorado de áreas lingüísticas (con especialidad en inglés) que, además de ser especialistas en la materia, ya ha dirigido TFG en inglés y sus alumnos han obtenido unos magníficos resultados en las defensas de sus TFG durante los cursos 2013/2014, 2014/2015 y 2015/2016.

b. Profesorado de áreas no lingüísticas (perteneciente a diferentes áreas de conocimiento). Este profesorado se identificó como un 
colectivo de profesionales que o bien han experimentado la dificultad de corregir un trabajo escrito en una lengua no materna (inglés), o bien prevé la dificultad de hacerlo en un futuro no lejano y, con esta preocupación (en el sentido más literal y etimológico de la palabra), se han acercado al profesorado especialista en busca de asesoramiento que les permita llevar a cabo esta tarea con la solvencia que una actividad de este tipo requiere.

c. Alumnado de Grado (perteneciente tanto a titulaciones de especialistas en segundas lenguas como a itinerarios bilingües) que durante el curso 2016/2017 estarán cursando la asignatura "Trabajo Fin de Grado" de su Titulación y que necesitan dominar las herramientas específicas de las redacciones de textos académicos y presentaciones orales en inglés. Destaca el alumnado del Grado en Educación Primaria, tanto en su itinerario bilingüe como de la mención en lengua extranjera: inglés.

d. Alumnado egresado y alumnado de Máster y de Doctorado de la UCO que han tenido la experiencia previa de redactar sus TFG, TFM o Tesis Doctorales en inglés y que pueden actuar de "mentores" para sus compañeros en esta tarea. La literatura especializada, así como la experiencia previa en otros proyectos de innovación docente en los que las coordinadoras de este proyecto hemos participado, nos dicen que la tutoría piramidal es una técnica metodológica que funciona e incide de manera positiva en el aprendizaje significativo del alumnado implicado.

\subsection{Materiales y método}

Las actividades que se realizaron a través de este Proyecto de Innovación Docente se resumen en las siguientes:

1. Celebración de un conjunto de seminarios escalonados en los que se trataron, discutieron y debatieron los aspectos esenciales de planificación, estructuración, técnicas de redacción y elaboración de sus presentaciones y defensas de los TFG en inglés.

2. Elaboración de una página web (http://www.uco.es/tfgeducacion/) donde se puede acceder a información y actividades complementarias y que sea accesible no solo para los participantes en el presente proyecto de innovación docente, sino también para el resto de alumnado de la Universidad de Córdoba, así como para cualquier otro miembro de la 
comunidad universitaria española que pudiera estar interesado, facilitando un mayor intercambio de ideas y una difusión de resultados más amplia.

3. Diseño y grabación de píldoras formativas (minivídeos docentes modulares) con la participación del profesorado implicado en el proyecto, así como del alumnado mentor, para explicar distintas partes de la elaboración de un TFG en inglés (siendo el compromiso mínimo la elaboración de cinco minivídeos durante todo el curso académico 2016/2017). Se trata de vídeos breves de entre 1 y 5 minutos de duración con los distintos elementos esenciales para el TFG, como los distintos tipos de TFG o cómo citar correctamente. Estos minivídeos están disponibles en la página web del proyecto, siempre en acceso abierto, a fin de que puedan consultarlos el conjunto del alumnado de la Universidad de Córdoba, así como cualquier persona interesada en la redacción de trabajos académicos en inglés a nivel global.

\section{DESCRIPCIÓN}

El desarrollo de este proyecto de innovación docente se ha llevado a cabo con dos grupos de actividades diferenciados, pero claramente relacionados entre sí. Por una parte, en el primer grupo de actividades contamos con los seminarios formativos desarrollados para la elaboración de Trabajos de Fin de Grado en inglés, sesiones que se han organizado para el alumnado del Grado en Educación Primaria, entre los que hay que destacar los casos del itinerario bilingüe y de la mención en lengua extranjera: inglés. Estos seminarios formativos, que en total han constado de seis sesiones temáticas, se recogen en la siguiente tabla (Tabla 1).

\section{Tabla 1. Seminarios formativos desarrollados en el proyecto de innovación docente}




\begin{tabular}{|c|c|}
\hline SEMINARS & CONTENTS \\
\hline \multirow{4}{*}{$\begin{array}{l}\text { Seminar 1: What is an } \\
\text { End-of-Degree } \\
\text { Dissertation? }\end{array}$} & Conceptualization \\
\hline & Different types of end-of-degree dissertations \\
\hline & Norms and rules of end-of-degree dissertations \\
\hline & Practical examples of end-of-degree dissertations \\
\hline \multirow{3}{*}{$\begin{array}{l}\text { Seminar 2: Searching } \\
\text { for Information }\end{array}$} & Key aspects when searching for information \\
\hline & Databases and resources \\
\hline & APA \\
\hline \multirow{3}{*}{$\begin{array}{l}\text { Seminar 3: English for } \\
\text { Academic Purposes in } \\
\text { End-of-Degree } \\
\text { Dissertations }\end{array}$} & Style and register \\
\hline & Theoretical foundations \\
\hline & $\begin{array}{l}\text { Structure and essential recommendations regarding } \\
\text { English for Academic Purposes }\end{array}$ \\
\hline $\begin{array}{l}\text { Seminar 4: } \\
\text { Conclusions }\end{array}$ & $\begin{array}{l}\text { How to write the conclusions of an end-of-degree } \\
\text { dissertation }\end{array}$ \\
\hline \multirow{2}{*}{$\begin{array}{l}\text { Seminar 5: } \\
\text { Presentation of the } \\
\text { End-of-Degree } \\
\text { Dissertation (I) }\end{array}$} & $\begin{array}{l}\text { Materials for the oral presentation of the end-of- } \\
\text { degree dissertation: poster, PPT, etc. }\end{array}$ \\
\hline & $\begin{array}{l}\text { Real examples of posters and presentations for end- } \\
\text { of-degree dissertations }\end{array}$ \\
\hline \multirow{3}{*}{$\begin{array}{l}\text { Seminar 6: } \\
\text { Presentation of the } \\
\text { End-of-Degree } \\
\text { Dissertation (II) }\end{array}$} & Planning and organising the presentation \\
\hline & Oral presentation \\
\hline & Facing potential questions \\
\hline
\end{tabular}

Fuente: Elaboración propia (en inglés para ajustarnos al desarrollo del proyecto)

Por otra parte, en el segundo grupo de tareas encontramos una serie de actividades necesarias para el desarrollo del trabajo, que se han llevado de manera interrelacionada entre los cuatro grupos de participantes implicados en el desarrollo del proyecto. El conjunto de estas actividades queda recogido en la Tabla 2.

Tabla 2. Actividades complementarias desarrolladas en el proyecto de innovación docente

\begin{tabular}{|l|l|}
\hline \multicolumn{1}{|c|}{ ACTIVIDADES } & \multicolumn{1}{|c|}{ CONTENIDOS } \\
\hline $\begin{array}{l}\text { Identificación de las principales } \\
\text { dificultades para elaborar los } \\
\text { TFG en inglés }\end{array}$ & $\begin{array}{l}\text { Principales dificultades; estructura del } \\
\text { TFG; inglés para fines académicos; } \\
\text { citación correcta en APA. }\end{array}$ \\
\hline $\begin{array}{l}\text { Organización y distribución } \\
\text { de los seminarios }\end{array}$ & $\begin{array}{l}\text { Número de sesiones; contenidos; } \\
\text { docentes implicados. }\end{array}$ \\
\hline $\begin{array}{l}\text { Grabación y edición de los } \\
\text { minivídeos docentes }\end{array}$ & $\begin{array}{l}\text { Grabación de vídeos con alumnado } \\
\text { que está desarrollando sus TFG y con } \\
\text { alumnado mentor; creación de vídeos } \\
\text { basado en infografías; edición de de }\end{array}$ \\
\hline
\end{tabular}




\begin{tabular}{|l|l|}
\hline $\begin{array}{l}\text { Creación y difusión de la } \\
\text { página web del proyecto }\end{array}$ & $\begin{array}{l}\text { vídeos para su posterior difusión. } \\
\text { Diseño, estructura y creación de la } \\
\text { página web asociada al proyecto, } \\
\text { dentro de la Universidad de Córdoba, a } \\
\text { fin de poder permitir el acceso libre y } \\
\text { gratuito de todas aquellas personas } \\
\text { interesadas en el proceso de } \\
\text { elaboración de un Trabajo de Fin de } \\
\text { Grado. }\end{array}$ \\
\hline $\begin{array}{l}\text { Participación en Congreso } \\
\text { Internacional para la difusión } \\
\text { del proyecto }\end{array}$ & $\begin{array}{l}\text { Participación en el 19th Annual } \\
\text { International Conference on Education } \\
\text { con la comunicación oral titulada "The } \\
\text { Use of Micro-Videos within Bilingual } \\
\text { Education", dentro del Panel titulado } \\
\text { "Bilingual Education: Opportunities } \\
\text { and Challenges". }\end{array}$ \\
\hline
\end{tabular}

\section{RESULTADOS OBTENIDOS}

Como ya avanzábamos en las secciones anteriores, esperamos obtener interesantes resultados relativos al nivel de lengua y las competencias que el alumnado participante obtenga.

Por otro lado, y sin menoscabo de los anteriores resultados, esperamos que el profesorado mejore su conocimiento sobre redacción textual en segundas lenguas, estructuración de los párrafos y la corrección de textos en inglés, al mismo tiempo que, esperamos, muestre mayor interés en la aceptación de la dirección de TFG en inglés en cursos venideros, ya que el profesorado no siempre muestra su predisposición a llevar a cabo una tarea en la que, por uno u otro motivo, encuentra dificultades que, creemos, podemos ayudar a salvar.

Al mismo tiempo, esperamos que la institución se beneficie de la mejora en la calidad de los TFG que se redactan en inglés ya que esto redunda en la calidad de los Títulos Universitarios, la futura empleabilidad de su alumnado, las tasas de rendimiento y, por supuesto, los resultados de calidad de la propia Universidad de Córdoba.

Este proyecto ha contado con los siguientes medios de difusión:

a. Página web (http://www.uco.es/tfg-educacion/): alojamiento de materiales, foros y recursos para el proyecto. Asimismo, se dispondrá de un repositorio de webs especializadas y materiales para el profesorado y alumnado participante. A su vez, al estar 
disponibles aquí los minivídeos elaborados para el proyecto, estos pueden compartirse a través de las redes sociales (YouTube, Twitter, Facebook, etc.).

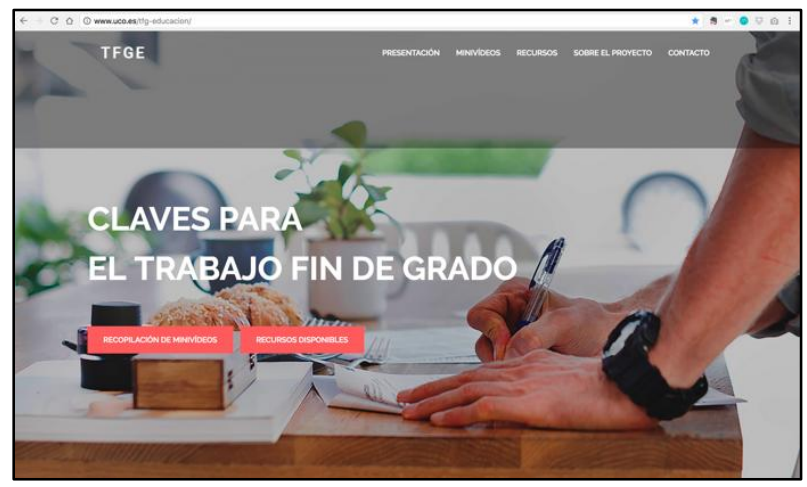

Fig. 1. Página principal de la web del Proyecto de Innovación Docente

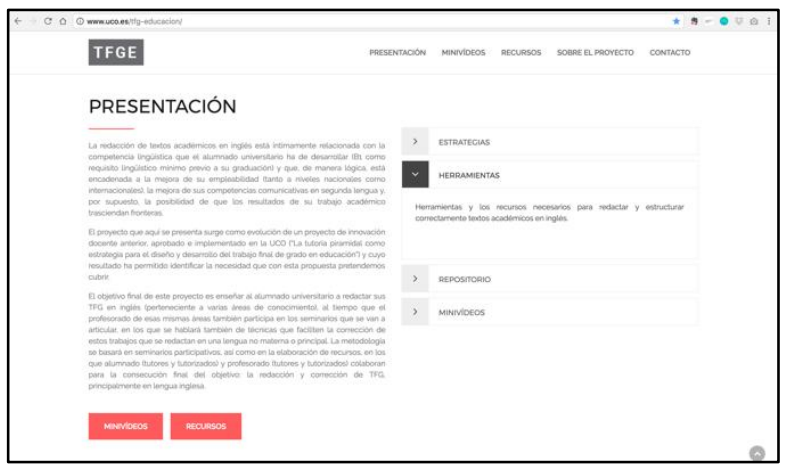

Fig. 2. Presentación del Proyecto

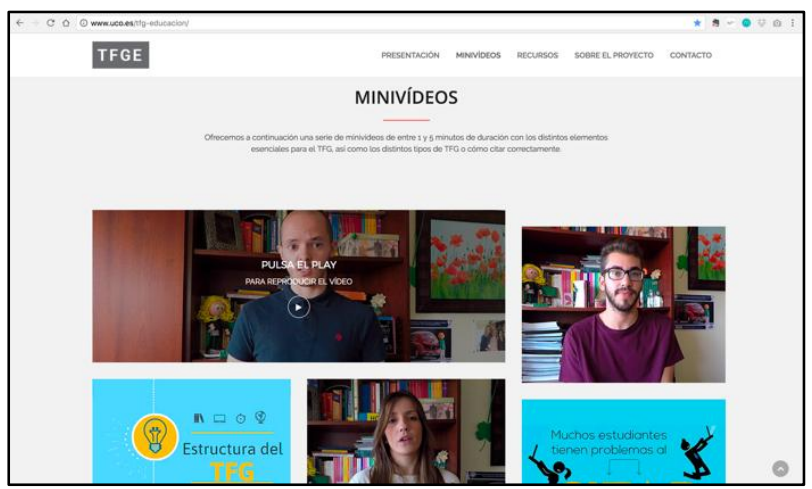


b. Difusión de determinados vídeos en YouTube, donde están disponibles las píldoras formativas (minivídeos docentes modulares).

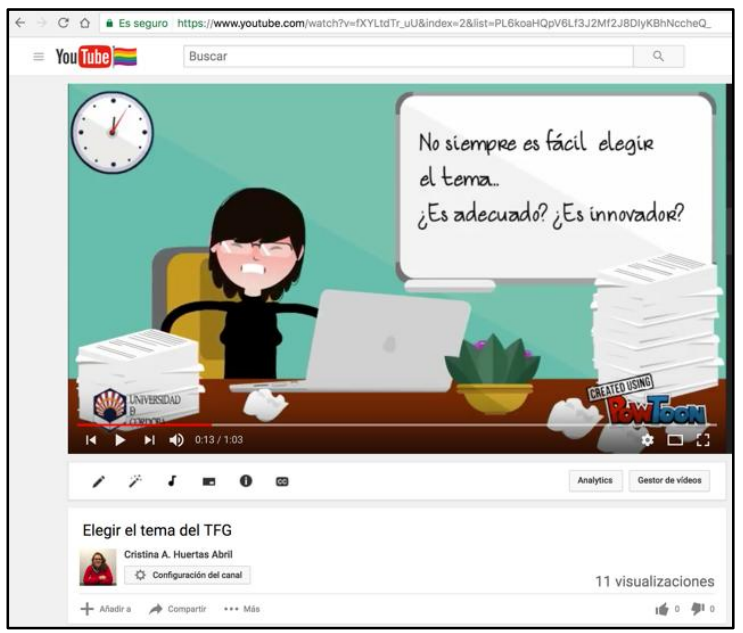

Fig. 4. Vídeos disponibles en YouTube

c. Participación en el Congreso Internacional 19th Annual International Conference on Education, ATINER, celebrado en Atenas (Grecia), con la comunicación oral titulada "The Use of Micro-Videos within Bilingual Education", dentro del Panel titulado "Bilingual Education: Opportunities and Challenges".

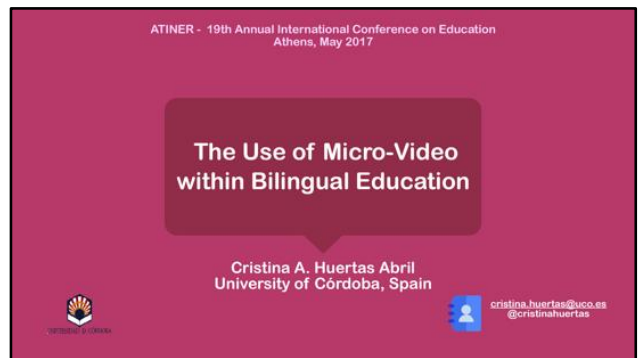

Fig. 5. Presentación en Congreso Internacional (selección 1) 


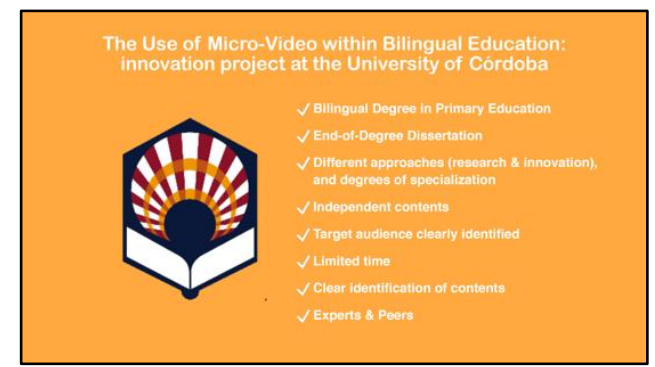

Fig. 6. Presentación en Congreso Internacional (selección 2)

\section{UTILIDAD/ANÁLISIS}

Esta experiencia didáctica (que continúa y complementa un proyecto de innovación docente financiado por la Universidad de Córdoba realizado durante el curso 2015/2016) ha favorecido el afianzamiento de un grupo de trabajo interdisciplinar, formado por cuatro colectivos claves del entorno universitario: profesorado de áreas lingüísticas, profesorado de áreas no lingüísticas, alumnado de Grado, y alumnado de Másteres y Doctorado. Esta diversidad de participantes ha favorecido el intercambio de ideas a nivel vertical (docentes-discentes) y a nivel horizontal (discentes-discentes y docentes-docentes).

Los procesos de enseñanza-aprendizaje para el desarrollo y la elaboración de Trabajos de Fin de Grado, principalmente en lengua inglesa, que se han establecido a ambos niveles han sido altamente beneficiosos para el conjunto del grupo. Esto ha permitido un mejor aprendizaje colaborativo, de manera que se han desarrollado tanto las distintas competencias como la mejora de la responsabilidad del aprendizaje propio como el de los compañeros del grupo de trabajo (Goikoetxea y Pascual, 2008).

De igual modo, el hecho de que los distintos participantes pertenecieran a distintas especialidades y tuvieran enfoques claramente diferenciados con respecto a los TFG (elaboración o redacción) permitió un intercambio de ideas de gran interés, lo cual ofreció un mayor enriquecimiento en el proceso formativo, así como la creación de sinergias. Entendemos que el proceso de peer tutoring (Topping, 2005; Bowman-Perrott, Davis, Vannest, Williams, Greenwood y Parker, 2013) o tutorización entre iguales se ha visto fomentado y favorecido por la interdisciplinariedad y las distintas interacciones a fin de alcanzar un objetivo común: obtener unos TFG de calidad. 
Finalmente, el aprendizaje cooperativo (Johnson y Johnson, 1991) facilita la creación de climas de trabajo favorecedores del intercambio de ideas, de modo que aumenta la autonomía, la iniciativa personal y la capacidad de resiliencia. Asimismo, y siguiendo a Trujillo (2002), "el aprendizaje cooperativo sirve para desarrollar las competencias lingüístico-comunicativas (Hirst y Slavik, 1990), pero también para mejorar las competencias sociales y cognitivas (...) además de ser una herramienta de integración de la lengua y los contenidos curriculares, ejercicio fundamental en contextos de L2 y LE (DeAvila, Duncan y Navarrete, 1987)" (p. 152).

\section{CONCLUSIONES/DISCUSIÓN}

En la sociedad del siglo XXI, es indudable la hegemonía de la lengua inglesa como lingua franca (Byram, 2004) o incluso como "lengua global" (Crystal, 2003). En este contexto, la realización del TFG supone que el alumnado ha de demostrar la adquisición de las competencias asociadas al Título, ya que es considerado un documento que acredita la culminación del proceso formativo y que, por tanto, habilita y abre la puerta al desempeño de una profesión. Entre estas competencias, se encuentra también la competencia lingüística (B1 en lengua extranjera como requisito lingüístico mínimo previo a su graduación) y que, de manera lógica, está vinculada a la mejora de su empleabilidad, de sus competencias comunicativas en una segunda lengua y la posibilidad de que los resultados de su trabajo académico trasciendan fronteras.

Asimismo, y siguiendo a Gómez (2016, pp. 52-53): “El proceso de escritura académica es diferente a cualquier otro tipo de escritura debido al contenido, a la estructura y, sobre todo, al lector de estos textos que, normalmente, es un especialista en el tema y cuyos conocimientos avezados en el mismo no harán sino añadir presión al proceso de escritura. Si a esto sumamos el hecho de que, en ocasiones, el alumno ha de usar el inglés para la redacción de estos textos, la dificultad y la complejidad del ejercicio es patente".

En este contexto, la elaboración del TFG requiere de supervisión y revisión por parte de un tutor o tutora que, en términos generales, hace el seguimiento del proyecto y asesora al estudiantado en cada una de las fases: elección del tema, revisión bibliográfica y fundamentación teórica, metodología, redacción de resultados y conclusiones, y defensa. Esta situación se dificulta cuando este proceso ha de realizarse total o parcialmente en lengua 
inglesa, por lo que evidenciamos que los resultados indican que la mayoría de los participantes de este proyecto de innovación docente aprecian favorablemente el intercambio de ideas, el asesoramiento entre áreas lingüísticas y áreas no lingüísticas, el aprendizaje cooperativo, y el asesoramiento y tutorización como herramienta de mejora de los procesos de enseñanza-aprendizaje, disminuyendo la inseguridad y la ansiedad de tener que utilizar una lengua extranjera.

Por todo ello, en el proceso formativo ha sido fundamental por una parte la realización de los seminarios, que permitían el intercambio de ideas y la resolución de dudas, y, por otro, la creación de un repositorio basado en la elaboración de recursos propios (principalmente píldoras formativas o minivídeos docentes modulares), disponibles en la página web del proyecto. Como conclusión final, consideramos que implicar a los cuatro grupos de participantes (profesorado de áreas lingüísticas, profesorado de áreas no lingüísticas, alumnado de Grado, y alumnado de Másteres y Doctorado) ha permitido la obtención de resultados satisfactorios y se considera, por tanto, una buena propuesta de trabajo para afrontar la elaboración de TFG total o parcialmente en lengua inglesa.

\section{AgRADECIMIENTOS}

Este Proyecto de Innovación Docente ha sido posible gracias al Plan de Innovación y buenas prácticas docentes (2016-2017) de la Universidad de Córdoba para el curso 2016/2017, concretamente en la Modalidad 1 "Proyectos de Innovación Docente". 


\section{BIBLIOGRAFÍA}

Bowman-PerrotT, L., Davis, H., VAnNest, K., Williams, L., GREENWOOD, C. \& PARKER, R. "Academic benefits of peer tutoring: A meta-analytic review of single-case research". School Psychology Review, 2003/42(1), p. 39-55.

BYRAM, M. Routledge Encyclopaedia of Language Teaching and Learning. Routledge, 2004.

CRYSTAL, D. English as a Global Language. Cambridge University Press, 2003.

GoikoetxeA, E. y PASCUAL G. "Aprendizaje cooperativo: bases teóricas y hallazgos empíricos que explican su eficacia". Educación XXI, 2002/5, p. 227-247.

GÓMEZ, M.E. "Redacción de textos académicos y expresión oral en inglés". Guía práctica para el trabajo de fin de grado en Educación. Graó, 2016, p. 51-66.

Gómez, M. E., Serrano, R. y Huertas, C. A. "Los Trabajos Fin de Grado (TFG) del área de Educación en el sistema universitario español". La educación SÍ importa en el siglo XXI. Síntesis, 2016.

JOHNSON, D. y JOHNSON, R. T. Learning mathematics and cooperative learning lesson plans for teachers. Edina, 1991.

TOPPING, K. J. Trends in peer learning. Educational Psychology, 2005/25(6), p. 631-645.

SÁEZ, F.T. Aprendizaje Cooperativo para la enseñanza de la lengua. Publicaciones, 2002/32, p. 147-162. 